\title{
Principles and guidelines for managing tooth wear: a review
}

\author{
Azouzi $\mathbf{I}^{1^{*}}$, Kalghoum $\mathrm{I}^{1}$, Hadyaoui $\mathrm{D}^{2 *}$, Harzallah $\mathrm{B}^{2}$ and Cherif $\mathrm{M}^{3}$ \\ ${ }^{1}$ DDM, Department of Fixed Prosthodontics, faculty of dental medicine, Monastir, Tunisia \\ ${ }^{2}$ Professor, Department of Fixed Prosthodontics, Faculty of dental medicine, Monastir, Tunisia \\ ${ }^{3}$ Professor and head of department, Department of Fixed Prosthodontics, Faculty of dental medicine, Monastir, Tunisia
}

\begin{abstract}
Non-Carious Tooth Surface Loss (NCTSL) is considered as a current issue of concern. It's management is challenging. Through this paper, the focus will be put on the different forms, locations and causes. Emphasis is placed on the importance of a thorough examination and indexing to assess the severity of tooth wear.

A systematic and integrated approach leads to favorable and predictable prognosis. We aim to provide clear recommendations on the best treatment strategy and simplified guidelines to succeed tooth wear management? considering the patient's expectations, the esthetic demand as well as the risk profile.

The patient should be fully informed. Restoration of severe tooth wear should ideally be delayed as long as possible.

The different treatment modalities will be discussed according to the localization and the severity of tooth wear.

The conservative, minimally invasive restoration, should first be considered.
\end{abstract}

\section{Introduction}

Non-Carious Tooth Surface Loss (NCTSL) also termed Tooth Wear (TW) is a current issue of concern to dental practitioners regarding the diagnosis, identification of the etiological factors, prevention, and execution of an adequate treatment $[1,2]$.

It is a physiological process that occurs as part of the aging process $[1,3]$, causing an enamel loss of between $28-30 \mu \mathrm{m}$ per annum as proved by Van't Spijker et al., in 2009 [4].

Whereas, in 2013, Bartlett et al confirmed that practitioners are often faced with patients suffering from Tooth Substance Loss (TSL). When it compromises tooth survival or when it is disproportionate to the presenting age, it is therefore, referred as to being pathological [5].

Indeed, various forms of TW that commonly occur are linked to: diet, bruxism, parafunctional activity and so on. This leads to the loss of hard tissues [6,7] because of the combination of mechanical and chemical processes.

TSL forms do not include trauma, or bacteria [2] or developmental disorders [8]; they are currently considered challenging when it comes to their management. (Figure 1)

\section{Tooth Wear prevalence}

The precise prevalence of TSL is hard to establish due to differences in assessment criteria complicated by coexisting factors [7].

As expected, all studies showed an increase in prevalence that goes with age $[9,6]$.

Van't Spijker et al. 2009 showed an increase in the prevalence of severe tooth wear in the adult population from $3 \%$ (at 20 years of age) to $17 \%$ (at 70 years of age [4].
In 2013, Bartlett et al. proved that $29 \%$ of the European adults aged 18-35 years old presented NCTSL [5].

As for the children and adolescents, authors have also noticed that they do not exhibit severe attrition [sh1], but there are indications of wear, mainly because of dental erosion $[10,11]$.

\section{Classification}

According to Grippo classification established in 1991, four types of surface loss have been identified and distinguished by the different causes [12].

\section{1) Attrition}

Attrition is defined as the wearing away of tooth substance (or restoration) due to tooth to tooth contact [9]

It can be mostly remarkable in patients with a vegetarian diet. It is generally associated with parafunctional activity. [3].

The wear from attrition may be localized on the occlusal surfaces of posterior teeth, the palatal surfaces of maxillary anterior teeth, the

Correspondence to: Hadyaoui D, Department of Fixed Prosthodontics, Faculty of dental medicine, Monastir, Tunisia, E-mail : dalendaresearch@gamil.com

Azouzi I, DDM, Department of Fixed Prosthodontics, faculty of dental medicine, Monastir, Tunisia, E-mail : inesazouzi1@gamil.com

Key words: Abrasion, Attrition, Abfraction erosion, Tooth wear, Prevention, Restorative treatment

Received: December 20, 2017; Accepted: January 26, 2018; Published: January 29,2018 


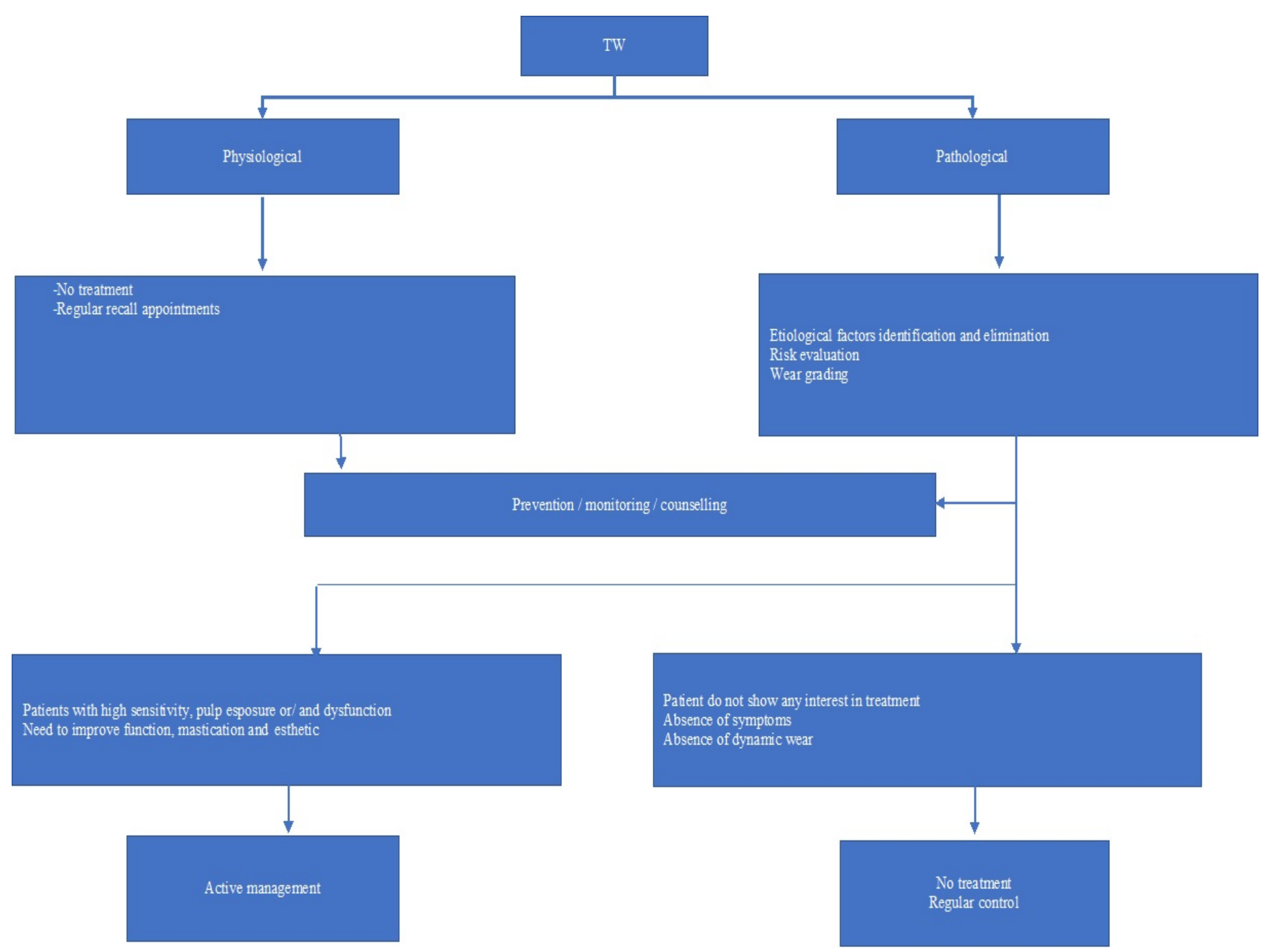

Figure 1. Flowchart of Tooth Wear (TW) management: to restore or not

labial surfaces of mandibular anterior teeth, and the incisal edges of anterior teeth. The affected surfaces are usually hard, smooth, and shiny. In certain cases, they may be sharp and jagged. The areas of attrition may be associated with a yellowish-brown discoloration if the wear has penetrated the enamel. Wear may also concern interproximal surfaces leading to mesial drifting and broadening of proximal contacts [6] (Figure 2).

\section{2) Abrasion}

Abrasion occurs when dental substance wears away due to friction between the tooth surface and external agent other than an opposing tooth [1].

Factors related to patients include abnormal process or habits [13]. Tooth brushing has been incriminated as to be the main etiological factor in dental abrasion [14]. This has been noted clinically, while observing a coincidence of smooth-surface and/or cervical abrasion as well as an extensive oral hygiene. This causes cervical abrasion where the severity depends on the brushing technique, time spent on brushing as well as the force applied on brushing [15].

Besides, the abrasion of interdental areas may be the result of extensive use of interdental brushes or tooth picks. [15].
Notching of incisal edges may be caused by pipe smoking, nuts and seed cracking, nail or thread biting, and hairpin biting which are rarely seen today $[13,15]$.

Other external factors include:

a) Material factors: they have been found to influence the prevalence of abrasion such as amount, $\mathrm{pH}$ and abrasiveness of tooth pastes used for brushing or inserted within interdental brushes. Similarly, tooth brush stiffness and their bristle design and flexibility -mainly when they are associated with aggressive brushing techniquesexplain this prevalence $[15,13]$.

As for restorations, unglazed porcelain causes abrasion of opposing natural teeth [16].

b) Environmental factors: they include exposure to dust and grit in some working places [17].

As with attrition, the condition is rarely caused by a single factor with erosion being a common cofactor $[3,18,19]$.

\section{3) Erosion}

The term erosion refers to NCTSL caused by chemical dissolution not related to acids produced by dental plaque. It may involve the 


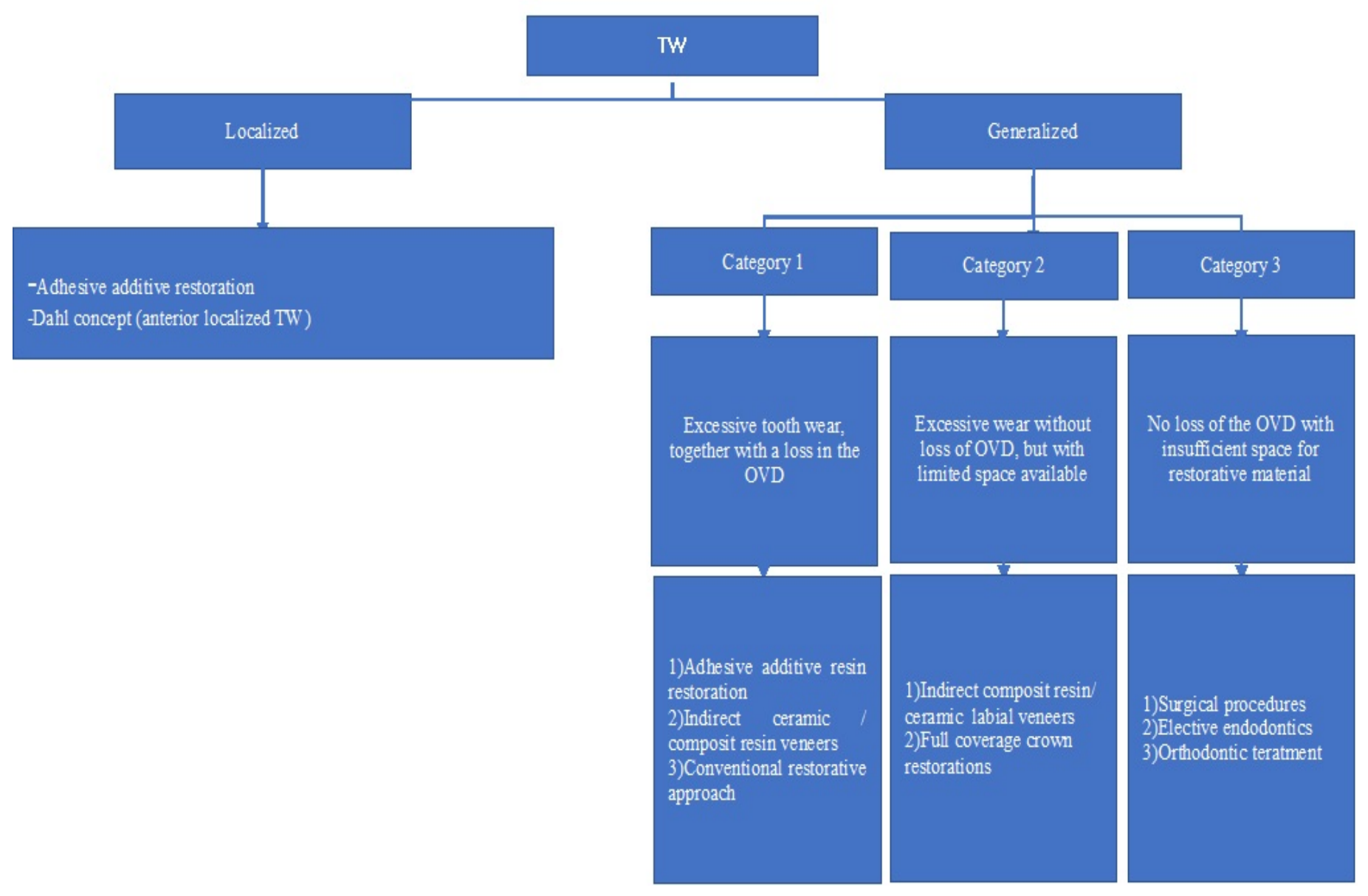

Figure 2. Flowchart of Active TW management

entire dentition or be localized as a single solitary lesion [6].

According to the characteristic pattern, two forms of erosion are to be considered:

The first erosion pattern is characterized by evident concave depressions where enamel appears thin and translucent. These are commonly exhibited on the palatal and occlusal surfaces of maxillary teeth as well as buccal and occlusal surfaces of mandibular posterior teeth. This specific pattern is linked to certain voluntary or involuntary medical conditions.

Voluntary or self-induced endogenous regurgitation of stomach contents is due to anorexic or bulimia nervosa. Involuntary regurgitation is explained by gastroesophageal reflux disease (GERD) or gastric ulcers and hiatus hernias.

It should be noted that lesions due to GERD are called perimolysis or perimylolysis $[13,3,1]$.

The second erosion pattern is specified by scooped out depressions present on labial surfaces of maxillary anterior teeth. [sh34, sh7] This is mainly caused by dietary factors, associated with slimness food and acidic drinks (fruit juices, carbonated or cola drinks, vinegar...) $[1,21]$.

Other exogenous factors such as exposure to corrosive or acidic substances and fumes that have a critical $\mathrm{pH}$ level of less than 5.5 (work in battery factories) have been reported. [22] Some sports as swimming in pools with low $\mathrm{pH}$ can also cause potential surface tooth loss [3].
Both patterns are caused by saucerized defects where acid is in contact with the teeth which is different from chemical dissolution not related to acid produced by dental plaque. These effects may be aggravated by the reduction in salivary flow caused by dehydration and some drugs [3].

\section{4) Abfraction}

Abfraction means the pathological loss of tooth structure resulting in wedge-shaped lesions with sharp line angles [Sh3, sh7] which may be located completely beneath the marginal gingiva [6].

Although these lesions have been recognized for years, their etiology has been debated. Numerous hypotheses were suggested to explain their exact cause [23].

Practitioners widely accept that abfraction is related to atypical occlusal loading [9,2].

It is a consequence of eccentric forces on the natural dentition, which were theoretically said to have been caused by tooth fatigue, flexure and deformation via biomechanical loading of the tooth structure. Cusp flexure, due to lateral occlusal forces during mastication and parafunctions, causes tensile stress. This disrupts the chemical bonds presented by induced cracks in the enamel and dentin in the region of concentrated stress at the cervical region. [1,24].

While some authors have shown evidence that supports this hypothesis, in 2006 Bartlett and Shah [26] suggested that such evidence is not that solid. They suggested that cervical lesions are 
probably accelerated when they are combined to erosion, abrasion, and attrition.

Abrasion in the cervical region is usually distinguishable from abfraction by its smooth, rounded nature of the lesion along with minimal to extreme gingival recession-with or without mucogingival defects. Gingival recession may also be seen with abfraction but is not a hallmark of these defects [6].

\section{NCTSL assessement}

Numerous indices have been proposed to assist the clinician in recording NCTSL.

No clear classification system has been presented yet.

The most popular index suggested is the Tooth Wear Index (TWI), by Smith and Knight in 1984 [27], which is primarily used in epidemiological studies. The TWI has been criticized for only relying on the ability of the clinician to visually identify exposed dentine.

This could potentially introduce interexaminer bias [1].

It does not include restored dentition. Nor does it relate the etiology to the outcome of the wear seen on the teeth. This makes it inaccurate to provide a complete picture of the clinical problem [28].

The Basic Erosive Wear Examination (BEWE), first described in 2008 by Bartlett et al. [29] is used to classify the patient's risk level for NCTSL. It is similarly annotated as the basic periodontal examination (BPE).

The BEWE describes NCTSL according to the measurable surface area affected rather than the depth of the lesion regarding dentine or pulpal exposure. The worst affected tooth for each sextant is examined. Hence, 0 (no wear), 1 (initial loss of surface texture), 2 (less than $50 \%$ loss of surface) and 3 (greater than $50 \%$ loss of surface). On completion of the BEWE, scores are summated towards a single grade.

Further classifications have been suggested as to provide a treatment guide to the practitioner.

The Anterior Clinical Erosive Classification (ACE) was proposed by Vailati and Belser in 2010 [sh39]. They established classification levels of wear in the palatal surface of the upper anterior teeth according to the severity of the dentine exposure as well as the preservation of enamel and tooth vitality.

The Tooth Wear Evaluation System (TWES) is a modular clinical guideline to optimize the diagnostic process (qualifying and quantifying). The surfaces playing a role in the occlusion and articulation are graded for each sextant using a 5-point ordinal occlusal/ incisal grading scale $(0=$ no wear; $1=$ wear confined to enamel; $2=$ wear with exposed dentin $\leq 1 / 3$ of crown height; 3 = wear $>1 / 3$ but $<2 / 3$ of crown height; 4 = wear $\geq 2 / 3$ of crown height)

Besides, because the palatal surfaces have a significant role in the articulation, the opposite sextant is also graded using a 3-point ordinal non-occlusal/ non-incisal grading scale

$(0=$ no wear; $1=$ wear confined to enamel; $2=$ wear with exposed dentin)

Each score is then singly recorded but not summated.

The TWES enables the clinician to judge, in advance, the degree of the desired plan difficulty and the appropriate decision considering the choice of the proper strategy of the treatment.
In general, indices serve as an aid to assess the extend and severity of tooth wear and can serve as indicators of a need for treatment.

A single indexing can be useful to quantify the severity of tooth wear, whereas, several recalls are needed to successively score the grade of tooth wear to determine or qualify whether it is progressive (dynamic) or not [30].

\section{Prevention strategies}

Admittedly, prevention of tooth surface loss is preferable to restoration.

To prevent TW aggravation, etiological factors must be determined along with early recognition of signs and symptoms. Thus, Prevention of further tooth wear ideally includes elimination of the causative agent. This simple action is often impossible, though [32].

Several researchers agree that wear progression can be reduced by patient education. This is based on preventive advice tailored to the cause and severity of the NCTSL. It focuses on:

\section{Dietary}

Patients are required to confine acidic drinks to meal times in favor of neutral drinks like milk and water along the day. (Bartlett et al., 2011) [5].

As for fruit juice consumption, it is preferable to be consumed once a day. The use of wide-bore drinking straws is highly recommended when consuming acidic beverages which helps to reduce the tooth-acid contact time. (Fung and Brearley Messer,2013) [33].

\section{Adequate tooth brushing: technique, brushing time and frequency}

It has been proved that dental tissues take 60 minutes to be reestablished after contact to acid, tooth brushing should not be performed soon after vomiting (Bartlett et al., 2013) [34] or after acidic drinking (Attin et al., 2001). [35].

\section{Fluoride addition}

It has been proved to reduce the damage of acid drinks to teeth. Whereas it's application, via mouth rinses gels is recommended to fight acid damage, requiring thus, the use of $0.7 \%$ fluoride solution followed by home application of $0.4 \%$ fluoride [36]. Other authors report that tooth pastes containing potassium are also considered to be appropriate for the management of sensitive dentine [30].

When it comes to parafunctional activity, patients with GERD are advised to wear a protective occlusal splint. However, specific attention and rigorous hygiene instructions are recommended as acidic substances may accumulate within the splints which may further exacerbate the rate of TW. [30]

Reference to other medical specialities is recommended in case of voluntary vomiting and suspected gastro-esophageal reflux [30].

\section{Patient's tress control}

It is key to tooth wear progression management. The previous methods based on advice and life style management are crucial to preserve the remaining tooth structure and to enhance the longevity of restorative treatment. Removing the etiological factors highly determines the treatment success. Whereas, failure can be early predicted if culprit factors couldn't be managed. 


\section{Clinical approach of worn dentition management}

There is a lack of detailed recommendations and specific guidelines for worn teeth treatment in literature.

The previously published recommendations are largely based on rigorous studies which are conventionally regarded as being of less scientific depth than randomized controlled trials and clinical experiments [37].

A systematic and integrated approach leads to favorable and predictable prognosis.

A thorough general and oral health history as well as the patient's emotional well-being and expectations are considered [38].

\section{Clinical examination}

An extraoral examination is necessary. [30] It involves palpation and auscultation of temporo mandibular joints (TMJ). The presence of muscle tenderness as well as clicking or crepitations in TMJ must be checked. The opening trajectory is examined and any deviation on opening or closure must be noted. Another important feature to be marked is the enlargement of the parotide gland which is a typical characteristic of bulimic patients [39].

Smile, lip line, midline discrepancies and facial vertical proportions should also be checked.

\section{Occlusal Vertical Dimension (OVD) evaluation}

It is critical to assess the loss of the OVD before any restoration. The different techniques used are: assessment of phonetic (sibilant sounds), the use of interocclusal distance, and the evaluation of soft tissue contours. The most commonly described method includes the foreway space evaluation, patients resting vertical dimension (RVD) and the use of calipers or Willis gauge $[37,30]$.

Dawson [40], Thompson [41] stated that a compensation of OVD loss by tooth eruption, alveolar bone expansion and muscle action could happen. This dento alveaolar compensation may help the OVD remain relatively constant or even increasing, despite the tooth wear: being a reason not to increase it as part of the reconstruction [M33].

Whereas, patients with OVD loss usually show overclosure and a typically inverted lip profile [30,37].

Intraoral examination: the quality of oral hygiene should be evaluated together with basic periodontal assessment. Any signs of xerostomia should be noted as well as the presence of buccal keratosis and tongue scalping.

Tooth vitality should also be assessed. Many sensitivity tests such as the application of ethyl chloride, warmed gutta percha or electric stimuli could be useful [30].

A cautious approach is advocated with patients exhibiting signs or symptoms of myofacial pain or disorder as jaw relationship records may not be accurate. These patients should first be treated with reversible methods such as interim occlusal splint to reduce the signs and symptoms of temporo mandibular disorders and normalize function before starting any prosthodontic therapy $[38,42]$.

The objectives of the treatment : [38]

(1) A comfortably functioning TMJ system

(2) An adherence to the basic principles of occlusion announced by Schuyler
(3) Restorations that will not violate the patient's neutral zone.

(4) Simultaneous stable bilateral tooth contacts in OMI

(5) A Centric Relation (CR) coinciding with Centric Occlusion (CO)

(6) A Mutually protected occlusion: anterior guidance that guarantees immediate disclusion of posterior teeth during eccentric movements

(7) Canine guidance: indeed, Hamburger et al. [43] have proved that patients with canine guidance have higher success rate than those without [38].

(8) Comfortable and asymptomatic temporo mandibular joints and stomatognathic musculature.

\section{Pretreatment phase}

Impressions must be performed then, and two complete sets of diagnostic casts be poured with die stone. A complete jaw relationship records must be made. Casts are mounted on the semi adjustable articulator. One set is maintained as an unaltered pretreatment record, while the other is modified by reconstructive wax up during diagnostic treatment planning. This blueprint is a pattern integrating a treatment plan that is properly sequenced and used for provisional restorations constructed to a new planned functional occlusion [30,38].

\section{Criteria for treatment decision}

Careful comprehensive treatment plan is required for each individual case [44].

Indexing should be applied to rate worn dentition. Nevertheless, more information is needed to indicate whether it is necessary to restore.

Generally, when patients do not show any interest in the treatment and in the absence of symptoms, a preventive approach seems to be more efficient- mainly when the patient doesn't present a dynamic wear process $[30,38]$.

However, for some other cases, restorative treatment may be required. Nevertheless, prosthetic rehabilitation in such patients is risky and prone to problems because of the complex structure of the stomatognathic system [45].

For those who show a high sensitivity and need to improve function, mastication and esthetic, restorative procedures become highly beneficial.

Some authors stress the benefit of delaying restorative treatment to an older age to avoid recycling the restoration procedure in case of failure.

Several treatment criteria have been suggested to guide the restorative treatment depending on the patient complaints, the amount of TSL, the affected teeth and surfaces (incisal, occlusal). These are correlated with the speed of tooth wear. Hence, a fast restorative treatment is required. The prosthodontist must make decisions regarding the need for treatment, the treatment procedure and the material. Moreover, progressive tooth wear may require a lifelong need for considerable maintenance [44].

According to TWES classification [31], only preventive measures and monitoring should be advocated when grade 0 or 1 exists on the surfaces that are involved in occlusion/articulation, and/or grade 0 or 1 on the non-occlusal/non-incisal surfaces. Similarly, restorative 
treatment is to be considered when grade 3 or 4 exists on the surfaces that are involved in occlusion/articulation, and/or grade 2 on the nonocclusal/non-incisal surfaces are diagnosed as well.

\section{Material choice}

\section{1/adhesive techniques}

Currently adhesive dentistry shows a considerable progress allowing advanced restoration techniques. Thus, many treatment options were proposed such as: direct/ indirect composite resin restorations, cast adhesive alloy (metal palatal veneers and onlays, and bonded ceramic restorations).

\section{Direct composite resin}

The use of composite resin to treat cases of tooth wear was first described by Bevenius et al. [47]

According to Briggs et al. [48] a rim of enamel, known as the 'gingival ring', is usually present at the gingival margins of severely eroded teeth. It enhances the predictability of bonding hard tissue to resin composites.

As mentioned by Poyser et al. [49], it is recommended that direct resin composite materials must be placed at a minimal increment thickness of 1.5 to $2.0 \mathrm{~mm}$ in all areas of functional loading to ensure appropriate longevity.

Benefits of direct composite resin in TW management [50,51,52].

- Acceptable esthetic outcome

- A conservative procedure applied with minimal or zero intervention

- Well tolerated by pulpal tissues

- Minimally abrasive to antagonistic tissues

- Acceptable quality / cost ratio

- Possibly applied in a single intervention

- Ease of restoration adjustment

- May serve as a tool to assess tolerance and adaptability to any altered occlusal and esthetic changes mainly for patients who can't tolerate planned changes to their occlusal schemes [50].

Drawbacks of direct resin composite restorations in TW management [51]:

- Need for good quality/quantity of dental enamel for effective adhesiveness.

- Polymerization shrinkage, which may lead to marginal micro-leakage and staining

- Possible bulk fracture(s)

- Need for optimal moisture control

- Limited control over occlusal and inter-proximal contours.

- Accelerated wear rate compared to metals/ceramics and potential inadequate wear resistance for posterior use

- Need for high rigor, particularly for palatal veneers.

Many placement techniques for composite resin restorations have been described [51, 52]: a direct technique termed the freehand application approach and two hemi direct ones using customized polyvinylsiloxane guide or thermoplastic vacuum formed matrix.

\section{1)The free hand technique}

This technique enables to conduct a purely additive treatment without affecting the sound hard tooth tissue. A highly skilled practitioner, being aware of the biomechanics principles, the concept of functional occlusion and esthetics, is more likely to conduct a treatment as such.

The resin is applied in bulk over the tooth surfaces. In a single visit, this technique is cost-effective.

It may be reliable in case of small number of worn dentition. However, when full arch rehabilitation is needed, the procedure is time consuming and totally depends on the operator's skills.

When the practitioner fails to assess the dynamic occlusal scheme, premature restorative flaw may occur.

\section{2) The hemi direct technique using a polyvinylsiloxane matrix guide}

This technique involves the use of duplicated waxed casts modified according to the desirable esthetic / function outcome. The matrix performed is used for direct composite layering.

This technique is criticized for involving major voids which end up to the need for copious adjustments. Hence, transparent silicones were suggested. They ensure the application of adequate quantities of material. Nevertheless, these silicones lack rigidity which may compromise their positioning and manipulation.

\section{3) The hemi direct using thermoplastic vacuum formed matrix guide}

The last described technique is using a customized vacuum formed matrix guide where a duplicate stone cast of the diagnosis waxed model is used to perform a translucent matrix of one mm thick, from a rigid material for accurate seating.

Relief holes to avoid air incrimination can be pierced. It is also recommended to place wedges to avoid bonding of resin material to interproximal surfaces.

Worn teeth must be conditioned and then resin is applied into the matrix precisely positioned and photopolymerized through the matrix.

Some authors suggested the use of preheated injected resin.

Despite the satisfying esthetic result it offers; this technique has numerous drawbacks ranging from poorly fitting matrices to overfilling ones which might culminate in gross excess along with air entrapment and potential post-operative sensitivity.

Milosovic (2015) [53] concluded that composite is a suitable restorative material for worn dentition with a low annual failure rate of $5.4 \%$. However, this failure was not associated with increased vertical dimension or bruxism but was significantly in older subjects and when a lack of posterior support was present.

Indirect resin restorations [51].

They are currently based on mechanically resistant hybrid resin when compared to micro fitted resins. They show an improvement of provisional surfaces wear while offering a better control of occlusal contour and vertical dimension, especially in the case of multiple restorations. In fact, dry heating treatment permits to eliminate internal porosities and evade oxygen incorporated in the resin which jeopardize polymerization. Also, it gives specific hardness and abrasion 
resistance to resin. Nevertheless, the indirect resin composite technique has numerous drawbacks. In fact, it requires at least two appointments. The hard tissue undercuts must be priorly removed, restorations might be bulky and inadequate wear resistance for posterior use may occur. Furthermore, the material used -resin composite- may have inferior marginal fit when compared to metal and ceramic. Add to that, the resin bonded luting agent may be exposed to wear and leakage.

Hamburger et al. [31] proved that whether for ceramic or resin composites, there is a significant positive relationship between layer thickness and compressive strength. Whereas, the choice between direct and indirect composite versus ceramic is specifically related to brands rather than material groups.

However, studies on the use of indirect ceramic and composite restorations in the management of severe tooth wear, lacks evidence and in-depth research.

\section{2/ Conventional fixed prosthodontics $[37,28]$}

It would still be the treatment of choice for rehabilitating extremely worn teeth. However, such treatment is complex and is still considered to be invasive.

Indeed, splinting to compensate short primary poorly retentive abutments is contra indicated. Full coverage crown usually requires provisional restorations that should be seated for enough time to refine and validate functionality of the occlusal design.

\section{3/ Adhesive versus conventional approach $[\mathbf{2 8 , 5 0}]$}

Compared to conventional restorative techniques which require removal of sound dental hard tissue, adhesive restorations are minimally invasive. They are described as the dynamic restorative concept.

There is no need for tooth preparation to guarantee the retention and the mechanical resistance will be offered using reliable materials where the resistance is increased by bonding.

Besides, conventional full coverage crowns may lead to the loss of tooth vitality where pulpal exposure is more likely to happen with teeth affected by wear process. Thus, adhesive additive approach might be primarily considered, when attempting to actively manage TW.

\section{Treatment modalities}

In case of the presence of pain, dysfunction and pulp exposure as well as esthetic demand, restorative treatments becomes a necessity despite being technically complicated.

So far, four restorative procedures have been described in terms of wear location maxillary anterior, mandibular anterior, localized posterior and generalized wear.

Other factors, including residual tooth structure, geography of surface loss, available quantity of enamel, lack of interocclusal clearance and esthetic demands of patients must be taken into consideration.

\section{Active restorative management of localized TW}

In case of localized tooth wear -especially in the anterior maxillary region associated with inadequate inter-occlusal space in either centric relation or centric occlusion- the Dhal concept is adopted. It is based on cobalt chromium removable appliance in the maxillary anterior region retained by clasps on canines and premolars to increase the vertical dimension of 2-3 $\mathrm{mm}$.
It is based on covering the cingulum areas of worn teeth leading thus to posterior teeth disclusion. This appliance is intraorally located until reestablished interocclusal contact between posterior teeth over a period of 4 to 6 months according to Dahl et al. [54]. According to Poyser et al., his period might be prolonged up to 18 to 24 months in some cases. [55] Thus, a space is created between anterior maxillary teeth and mandibular dentition. Preparation of anterior teeth becomes, thereby, needless in favor of a non- invasive restoration.

Despite the success rate reported by Poyser et al. [55] (94\% to 100\%) and Gulamali et al. [56] (5.8 years) considering Dahl concept,

Hemmings et al. [57] proved its limitations in case of patient with mal occlusion (cl III or mandibular facial asymmetry) or with patients showing limited eruptive potential (dental implants, anterior open bite...).

Due care has to be given also to patients with symptomatic TMJ or presenting periodontal disease or in post orthodontic treatment as well as among patients under oral or intravenous bisphosphonate treatment [58].

\section{Active restorative management of generalized TW: $[21,50]$}

Turner and Missirilian considered three categories of generalized TW patients:

Category 1: Excessive tooth wear, together with a loss in the OVD

Category 2: Excessive wear without loss of OVD, but with limited space available

Category 3: No loss of the OVD with insufficient space for restorative material

Here, the TW management varies.

\section{Category 1: Excessive tooth wear, together with a loss in the OVD}

They are the most straightforwardly managed. The required space for restorative material is already cleared through the tooth wear process. Thus, the increase in OVD is expected without any occlusal reduction. This can be applied using an acrylic stabilization splint enabling to evaluate the patient's ability to accept changes according to planned occlusal schemes. The splint will ovoid not only an increase in the OVD but also offers a mutually protective occlusal scheme.

However, for some authors, the use of this appliance for these patients is not essential. [58]

The increased ODV is equally divided between maxillary and mandibular arches and this to guarantee a successful adaptation and the reduction of the OVD increasing abruptness.

Obviously, there are two approaches to adopt: the adhesive additive and the conventional restorative approach. During the former, all the involved teeth can be prepared in one visit: this preparation consists of finishing lines accommodation placement of adhesive restoration providing, thus, adequate resistance. The latter requires teeth preparations during a single visit.

Preparation of maxillary or mandibular teeth is performed according to the importance of tooth wear. In fact, arch where tooth wear pattern is more compromising the occlusal plane, is prepared first. Interim restorations will be performed to correct the occlusal plane discrepancies according to the planned OVD on the waxed casts used to perform silicone guides. 
They are seated for 6 to 8 weeks, the period necessary for assessing esthetic and function.

Once validated, definitive crowns are performed respecting the predetermined occlusion scheme.

\section{Category 2: Excessive wear without loss of OVD, but with limited space available}

In such cases, an existing discrepancy may be noted between $\mathrm{CO}$ and CR. The latter, may provide space to accommodate restorative material. However, this space might not always be fully adequate. Hence, the need for an OVD increase.

These patients are appropriate candidates for full coverage restorations.

The adaptability to the new OVD and the mutually protective scheme are evaluated by a splint continuously worn, except when eating, during a month. [59]

However, Vialati and Belser (2008) [60], suggested an alternative approach using indirect resin composite onlay and/ or palatal resin veneers. This approach has been criticized for being somehow irreversible as some level of tooth reduction may be involved accommodating the adequate thickness of composite resin. It is also time consuming and skill demanding. Furthermore, attaining the desired dynamic occlusal scheme particularly for complex posterior restorations may be difficult and even impossible to reach.

\section{CATEGORY 3 No loss of the OVD with insufficient space for restorative material}

These are the most complex cases to manage where no adequate space for restoration is available while compensatory tooth repositioning is observed.

The challenge faced in this situation is to provide space without increasing the OVD which is not evident.

A variety of strategies have been presented in literature:

-surgical procedure: it consists of crown lengthening with an osseous re-contouring increasing, thus, the height of coronal tooth tissue. This improves retention and resistance of the future conventional restoration. The shortcomings of the procedure being inadequate crown to root ratio, black triangles possible recessions and post-operative sensitivity in relation with newly exposed dentine.

-Elective endodontics may also be considered. The application of post and core reconstructions mainly to increase the height of the core and increase, thus, the retention of the future restorations. Nevertheless, success is partially dependent on endodontic treatment.

A less common strategic management for these typically challenging situations is the orthodontic treatment. It consists of intruding over erupted teeth or extruding short crowns associated with later restorative treatment. However, this option is complicated regarding time, costs and patient availability.

Unless such methods fail to provide enough space, an increase in the OVD would be promoted. It would be assessed through occlusal splints.

\section{Conclusion}

Despite the recommendations outlined for managing tooth wear, a lack of evidence is remaining. A rigorous clinical examination as well as a well rationalized diagnosis are keys to treatment success.
Planification must be based on treatment goals, which are initiated by preventive approaches and finalized by restorative treatment in cases of extensive tooth wear or esthetic demand. Minimally invasive procedures are invading the field of restorative management essentially based on the performance of adhesive agents. However, ultimate need for full coverage crowns is still imposed in specific situations of generalized severe tooth wear.

\section{References}

1. Alhilou A, Beddis HP, Mizban L, Seymour DW (2015) Basic Erosive Wear Examination: assessment and prevention. Dental Nursing 11:262-267. [Crossref]

2. Haddadin K, Rassas E, Masarweh N, Haddadin KH (2015) Causes for tooth surface loss in a group of Jordanian population. Pakistan Oral \& Dental Journal 35.

3. Hobkirk JA (2006) Tooth surface loss: causes and effects. The International journal of prosthodontics 20:340-341.

4. Spijker AV, Rodriguez JM, Kreulen CM, Bronkhorst EM, Bartlett DW, et al. (2009) Prevalence of tooth wear in adults. International Journal of Prosthodontics (1)

5. Bartlett DW, Fares J, Shirodaria S, Chiu K, Ahmad N, et al. (2011) The association of tooth wear, diet and dietary habits in adults aged 18-30 years old. Journal of dentistry. 39:811-816. [Crossref]

6. Curtis Jr JW, Farley BA, Goldstein RE (2002) Abfraction, Abrasion, Attrition and erosion. Esthetics in Dentistry. BC Decker Inc Hamilton, London 2:501-523.

7. Al-Zarea BK (2012) Tooth surface loss and associated risk factors in northern saudi arabia. ISRN dentistry.

8. Hattab FN, Yassin OM (2000) Etiology and diagnosis of tooth wear: a literature review and presentation of selected cases. International Journal of Prosthodontics 13.

9. Wood I, Jawad Z, Paisley C, Brunton P (2008) Non-carious cervical tooth surface loss: a literature review. Journal of dentistry 36:759-766. [Crossref]

10. Bartlett DW (2005) The role of erosion in tooth wear: aetiology, prevention and management. Int Dent J 55 (Suppl 1):277-284. [Crossref]

11. Jaeggi T, Lussi A (2006) Prevalence, incidence and distribution of erosion. Monogr Oral Sci 20:44-65. [Crossref]

12. Grippo JO (1991) Abfractions: a new classification of hard tissue lesions of teeth Journal of Esthetic Dentistry 3:14-19.

13. Litonjua LA, Andreana S, Bush PJ, Cohen RE (2003) Tooth wear: attrition, erosion, and abrasion. Quintessence Int 34:435-446.

14. Levitch LC, Bader JD, Shugars DA, Heymann HO (1994) Non-carious cervical lesions Journal of Dentistry 22:195-207.

15. Imfeld T (1996) Dental erosion. Definition, classification and links. European journal of oral sciences 104:151-155.

16. Mahalick JA, Knap FJ, Weiter EJ (1971) Occlusal wear in prosthodontics. J Am Dent Assoc 82:154.

17. Turner KA, Missirlian DM (1985) Restoration of the extremely worn dentition. $J$ Prosthet Dent 52:467-474.

18. Smith BG (1989) Tooth wear: Aetiology and diagnosis. Dent Update 16:204-212.

19. Smith BG, Knight K (1984) A comparison of patterns of tooth wear with aetiological factors. Br Dent J 157:16-19.

20. Eccles JD (1982) Erosion affecting the palatal surfaces of upper anterior teeth in young people. Br Dent J 152:375-378.

21. Krishna MG, Rao KS, Goyal K (2005) Prosthodontic management of severely worn dentition: including review of literature related to physiology and pathology of increased vertical dimension of occlusion. The Journal of Indian Prosthodontic Society $5: 89$.

22. Petersen PE, Gormsen C (1991) Oral conditions among German battery factory workers. Community Dent Oral Epidemiol 19:104-106.

23. Ghai KS, Gupta G, Goyal M (2013) The Management of Worn Dentition-A Systematic Approach. Indian Journal of Dental Sciences 5.

24. Grippo JO, Simring M, Coleman TA (2012) Abfraction, Abrasion, Biocorrosion, and the Enigma of Noncarious Cervical Lesions: A 20-Year Perspective. Journal of Esthetic and Restorative Dentistry 24:10-23. 
25. Braem M, Lambrechts P, Vanherle G (1992) Stress induced cervical lesions. J Prosthet Dent 67:718-722.

26. Bartlett DW, Shah P (2006) A critical review of non-carious cervical (wear) lesions and the role of abfraction, erosion and abrasion. J Dent Res 85:306-312.

27. Smith BG (1984) An index for measuring the wear of teeth. Br Dent J 156:435-438.

28. Loomansa B, Opdamb N, Attinc T, Bartlettd D, Edelhoffe D, et al. (2017) Severe Tooth Wear: European Consensus Statement on Management Guidelines. J Adhes Dent 19:111-119

29. Vailati F, Christoph Belser U (2010) Classification and treatment of the anterior maxillary dentition affected by dental erosion: the ACE classification. The The International Journal of Periodontics \& Restorative Dentistry 30:559.

30. Mehta SB, Banerji S, Millar BJ, Suarez-Feito JM (2014) Current concepts on the management of tooth wear: part 1. Assessment, treatment planning and strategies for the prevention and the passive management of tooth wear. Br Dent J 212:17-27. [Crossref]

31. Wetselaar P, Lobbezoo F (2016) The tooth wear evaluation system (TWES): a modular clinical guideline for the diagnosis and management planning of worn dentition. $J$ Craniomandibular Funct 8:313-326.

32. Wickens JL (1999) Tooth surface loss: Prevention and maintenance. Br Dent J 186:371-376.

33. Fung A, Messer LB (2013) Tooth wear and associated risk factors in a sample of Australian primary school children. Australian dental journal 58:235-245. [Crossref]

34. Bartlett DW, Lussi A, West NX, Bouchard P, Sanz M, et al. (2013) Prevalence of tooth wear on buccal and lingual surfaces and possible risk factors in young European adults. Journal of dentistry 41:1007-1013. [Crossref]

35. Attin T, Knöfel S, Buchalla W, Tütüncü R (2006) In situ evaluation of different remineralization periods to decrease brushing abrasion of demineralized enamel. Caries research 35:216-222. [Crossref]

36. Thrash W, Dodds M, Jones D (1994) The effects of stannous fluoride on dentinal hypersensitivity. Int Dent $J$ 1: 107-118.

37. Johansson A, Johansson AK, Omar R, Carlsson GE (2008) Rehabilitation of the worn dentition. Journal of oral rehabilitation 35:548-566. [Crossref]

38. Ghai KS, Gupta G, Goyal M (2013) The Management of Worn Dentition-A Systematic Approach. Indian Journal of Dental Sciences 5.

39. Milosevic A, Jones C (1996) Use of resin bonded ceramic crowns in a bulimic patient with severe erosion. Quintessence Int 1996; 27: 123-127.

40. Dawson PE (1989) Evaluation, diagnosis, and treatment of occlusal problems. Mosby Inc.

41. Thompson JR (1946) The rest position of the mandible and its significance to dental science. Journal of American Dental Association 33:151-180. [Crossref]

42. De Boever JA, Carlsson GE, Klineberg IJ (2000) Need for occlusal therapy and prosthodontic treatment in the management of temporomandibular disorders. Part II. Tooth loss and prosthodontics treatment. J Oral Rehabil 27:647-659. [Crossref]

43. Hamburger JT, Opdam NJ, Bronkhorst EM, Kreulen CM, Roeters JJ, et al. (2011) Clinical performance of direct composite restorations for treatment of severe tooth wear. J Adhes Dent 13:585-593.
44. Kaur S, Singh K, Suman N, Kaur M (2014) Management of severely worn dentition-a review. Indian Journal of Comprehensive Dental Care 4:67-69.

45. Cura C, Saraçoğlu A, Öztürk B (2002) Prosthetic rehabilitation of extremely worn dentitions. Quintessence Int 33:225-230.

46. Schuyler CH (1963) The function and importance of incisal guidance in oral rehabilitation. The Journal of Prosthetic Dentistry :1011-1029. [Crossref]

47. Bevenius J, Evans S, L'Estrange P (1994) Conservative management of erosionabrasion. A system for the general practitioner. Aust Dent J 39: 4-10. [Crossref]

48. Briggs P, Djemal S, Chana H, Kelleher M (1998) Young adult patients with established dental erosion - what should be done? Dent Update 25: 166-170.

49. Poyser N, Porter R, Briggs P, Kelleher M (2007) Demolition experts: managemen of the parafunctional patient: 2. Restorative management strategies. Dent Update 34: 262-268. [Crossref]

50. Mehta SB, Banerji S, Millar BJ, Suarez-Feito JM (2012) Current concepts on the management of tooth wear: part 2. Active restorative care 1: the management of localised tooth wear. Br Dent J 212:73-82. [Crossref]

51. Mehta SB, Banerji S, Millar BJ, Suarez-Feito JM (2012) Current concepts on the management of tooth wear: part 4 . An overview of the restorative techniques and dental materials commonly applied for the management of tooth wear. Br Dent J $212: 169-77$. [Crossref]

52. Mehta, Shamir B, Francis S, Banerji S (2016) A Guided, Conservative Approach for the Management of Localized Mandibular Anterior Tooth Wear. Dent Update 43:106108. [Crossref]

53. Milosevic A, Burnside G (2016) The survival of direct composite restorations in the management of severe tooth wear including attrition and erosion: a prospective 8-year study. J Dent 44:13-19. [Crossref]

54. Dahl B, Krungstad O, Karlsen K (1975) An alternative treatment of cases with advanced localised attrition. J Oral Rehabil 2: 209-214. [Crossref]

55. Poyser N, Porter R, Briggs P, Chana H, Kelleher M (2005) The Dahl concept: past, present and future. Br Dent $J$ 198: 669-676. [Crossref]

56. Gulamali AB, Hemmings KW, Tredwin CJ, Petrie A (2011) Survival analysis of composite Dahl restorations provided to manage localised anterior tooth wear (ten-year follow-up). Br Dent J 211: E9.[Crossref]

57. Hemmings K, Darbar U, Vaughn S (2000) Tooth wear treated with direct composite at an increased vertical dimension: results at 30 months. J Prosthet Dent 83: 287-293. [Crossref]

58. Mehta SB, Banerji S, Millar BJ, Suarez-Feito JM (2012) Current concepts on the management of tooth wear: part 3. Active restorative care 2: the management of generalised tooth wear. Br Dent J 212:121-127. [Crossref]

59. Rivera-Morales W, Mohl N (1993) Restoration of the vertical dimension of the occlusion in the severely worn dentition. Dent Clin North Am 36: 651-663.

60. Vialati F, Belser C (2008) Full mouth adhesive rehabilitation of a severely eroded dentition: The three-step technique. Part 2. Eur J Esthet Dent 3: 128-146.

Copyright: (C2018 Azouzi I. This is an open-access article distributed under the terms of the Creative Commons Attribution License, which permits unrestricted use, distribution, and reproduction in any medium, provided the original author and source are credited. 